Article

\title{
The PAK Inhibitor PF-3758309 Promotes the Inhibitory Effects of Multiple Chemotherapeutic Reagents on Patient- Derived Pancreatic Cancer Cell Lines
}

Kai Wang ${ }^{\S 1}$, Nhi Huynh ${ }^{\S 1}$, Xiao Wang' ${ }^{1}$, Marina Pajic ${ }^{2,3}$, Ashleigh Parkin², Jennifer Man², Graham S. Baldwin ${ }^{1}$, Hong $\mathrm{He}^{1 *}$ and Mehrdad Nikfarjam ${ }^{1 *}$

1. Department of Surgery, University of Melbourne, Austin Health, Studley Road, Heidelberg, Victoria 3084, Australia

2. The Kinghorn Cancer Centre, The Garvan Institute of Medical Research, 384 Victoria St, Darlinghurst, Sydney, NSW 2010, Australia

3. St Vincent's Clinical School, Faculty of Medicine, University of NSW, Australia

$\S$ These two people have made equal contributions to this paper.

* To whom correspondence should be addressed: Dept. of Surgery, University of Melbourne, Austin Health, Studley Rd., Heidelberg, Victoria 3084, Australia

Tel.: 6139496 5468; Fax: 6139458 1650;

Email: hong.he@unimelb.edu.au; $\underline{\text { m.nikfarjam@unimelb.edu.au }}$

\begin{abstract}
Pancreatic ductal adenocarcinoma (PDA) remains the most lethal malignancy due to lack of an effective treatment. P21-activated kinases (PAKs) play key roles in PDA growth, and the PAK inhibitor PF-3758309 synergistically reduced PDA growth with gemcitabine. The aim of this study was to determine the effect of PF-3758309 with multiple chemotherapeutic reagents on a panel of patient-derived PDA cell lines. Cells were treated with PF-3758309 plus or minus gemcitabine, 5fluorouracil (5-FU) or abraxane, and cell proliferation was determined. Protein expression profiles were measured by Western blot. PDA cells were subcutaneously injected into the flanks of SCID mice which were then treated with PF-3758309, gemcitabine, PF-3758309 plus gemcitabine, or gemcitabine plus abraxane. Tumour growth was measured by volume and weight. PF-3758309 enhanced the inhibitory effects of 5-FU, gemcitabine and abraxane on a panel of patient-derived PDA cells, inhibited HIF- $1 \alpha$ protein expression and reduced the protein levels of palladin and $\alpha$-SMA in these cells. The combination of PF-3758309 with gemcitabine maximally inhibited PDA growth in vivo, which was comparable to the combination of gemcitabine with abraxane. PF-3758309 enhanced the suppressive effects of multiple chemotherapeutic reagents on the growth of a panel of patientderived PDA cell lines. The combination of PF-3758309 with gemcitabine provides a potential treatment option with less toxicity than gemcitabine plus abraxane.
\end{abstract}

Keywords: p21-activated kinase (PAK); Pancreatic ductal adenocarcinoma (PDA); PF-3758309; gemcitabine

\section{Introduction}

The low survival rate of pancreatic ductal adenocarcinoma (PDA) is largely caused by resistance to chemotherapy. Because of the late diagnosis of PDA only $20 \%$ of patients are suitable for surgical resection, which makes chemotherapy a critical component in the clinical management of PDA. Gemcitabine has been the mainstay of systemic treatment for most stages of PDA. With the discovery 
of active multi-agent chemotherapeutic regimens, such as FOLFIRINOX (a combination of leucovorin (folinic acid), fluorouracil (5-FU), irinotecan and oxaliplatin) and gemcitabine plus nab-paclitaxel (abraxane), the treatment landscape of PDA is slowly evolving. FOLFIRINOX and gemcitabine plus abraxane are now utilized as standard first line treatment options in metastatic PDA [1]. FOLFIRINOX treatment offers a median survival of 11.1 months in patients with advanced disease [2], while gemcitabine plus abraxane treatment provides a median survival of 8.5 months [3]. Compared to gemcitabine monotherapy, the survival rate of PDA patients has been marginally improved with the combination treatment.

The unique desmoplastic stroma present in PDA forms a barrier of fibrotic tissue which prevents the penetration of chemotherapeutic reagents and thus compromises chemotherapy [4]. The stroma contains a large number of pancreatic stellate cells (PSCs), which are characterized by the expression of desmin, glial fibrillary acidic protein (GFAP), vimentin and nestin [5]. In pancreatic cancer, activated PSCs, expressing high level of $\alpha$-smooth muscle actin ( $\alpha$-SMA), play an important role in tumour-stroma interactions. Palladin, an actin binding protein, has been detected in both PDA cells and stroma, and the stromal expression of palladin is an independent prognostic factor in PDA and a surrogate indicator for the effectiveness of chemoradiation therapy [6].

P21-activated kinases (PAKs), acting downstream of KRas, play important roles in PDA development. PAKs can be divided into 2 groups, group I contains PAKs 1-3 and group II PAKs 4-6. Over-expression of PAK1 [7] and PAK4 [8] has been reported in PDA patients, and both PAK1 and PAK4 are involved in the proliferation and migration/invasion of PDA cells [9]. Inhibition of PAK1 synergistically with gemcitabine reduced the growth of pancreatic cancer cells in vitro and in vivo [10], and activation of PAK1 contributed to gemcitabine resistance of pancreatic cancer cells [11]. Inhibition of PAK1 also suppressed PSC activation and proliferation while promoting apoptosis, and deletion of stromal PAK1 increased survival of mice with PDA [12]. PAK4 promoted PDA cell proliferation and survival by activation of the NF- $\kappa B$ pathway [13], and inhibition of PAK4 increased the sensitivity of PDA cells to gemcitabine [14]. Inhibition of PAK4 decreased PDA cell proliferation via down-regulation of Bad phosphorylation and up-regulation of tumour suppressive miRNA [15]. A novel PAK4 allosteric inhibitor suppressed PDA cell proliferation on its own or synergistically with gemcitabine or oxaliplatin while promoting the apoptosis of PDA cells [16]. These effects of PAKs on PDA cells and stromal cells make PAKs attractive targets in PDA treatment.

The above data also indicates the importance of targeting both PAK1 and PAK4 in the treatment of PDA. The affinity of PF-3758309, a pan-PAK inhibitor, is highest for PAK4 (IC 50 2.7-4.5 nM), and second highest for PAK1 ( $\mathrm{IC}_{50} 14 \mathrm{nM}$ ) [17]. PF-3758309 suppressed the proliferation and migration/invasion of melanoma and lung cancer by targeting both PAK1 [18] and PAK4 [19]. PF3758309 inhibited the growth of colorectal cancer cells in vitro and in vivo by decreasing the activity of PAK1, and enhanced the inhibitory effect of 5-FU on the proliferation and tumorigenesis of colorectal cancer cells [20]. The aims of the current study were to determine the effects of PF-3758309, in combination with multiple chemotherapeutic reagents, on a panel of patient-derived PDA cell lines, and to define the possible mechanisms involved.

\section{Results}

PAK1 expression correlated with CK19; PAK4 expression with $\alpha$-SMA, palladin and GFAP

The protein expression profiles of six TKCC cell lines were determined by Western blots. The expressions (Fig.1 A) and relative levels (Fig.1B) of total and phosphorylated (active) PAK1 and PAK4, CK19, $\alpha$-SMA, palladin, GFAP and desmin were shown in Fig.1. Regression analysis showed that the protein expression of PAK1 was positively correlated with the protein expression of CK19, but not with $\alpha$-SMA, palladin, GFAP or desmin (Fig. 1C). However, the protein level of PAK4 was 
positively correlated with $\alpha$-SMA, palladin and GFAP, but not with CK19. This result indicates that PAK1 and PAK4 function differently in these patient-derived cancer cell lines.
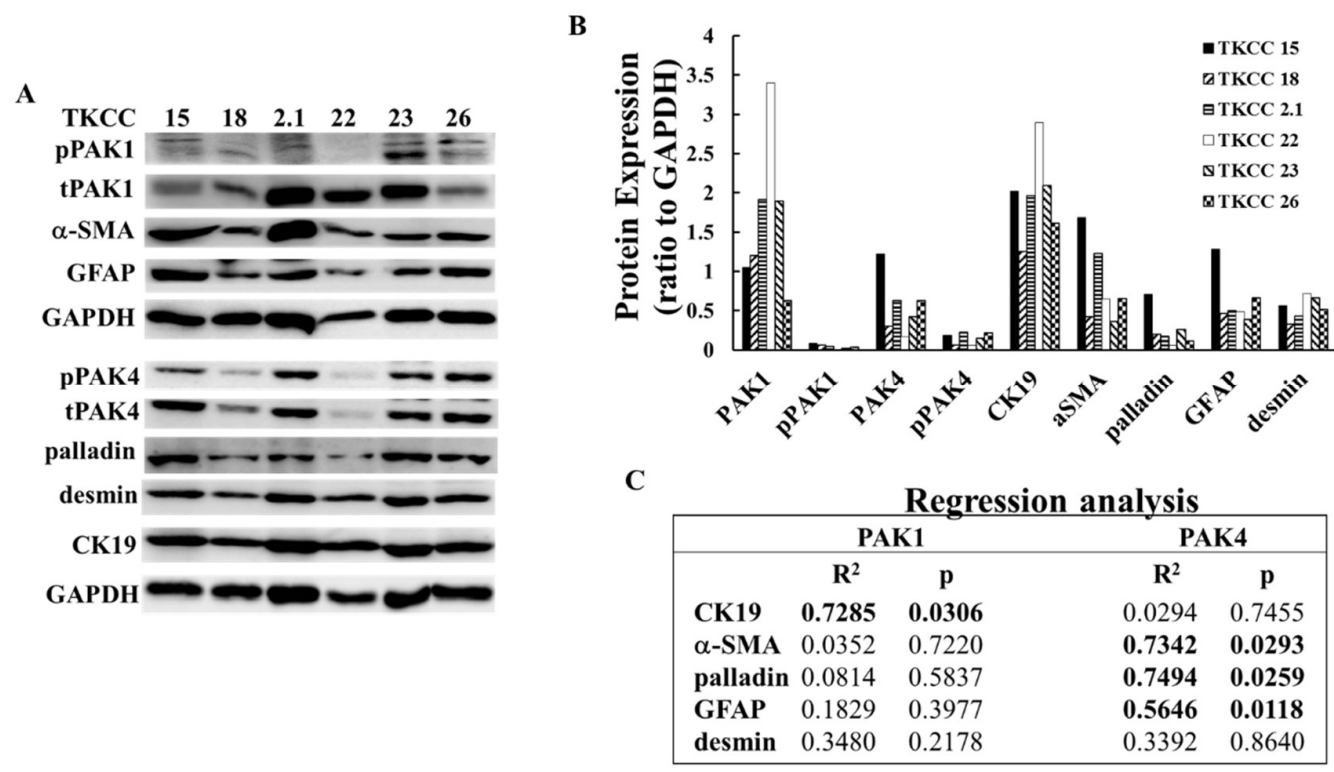

Fig.1. PAK1 expression correlated with CK19, while PAK4 expression correlated with $\alpha-S M A$, palladin and GFAP. The expression of several proteins in six TKCC cell lines was determined by Western blot (A), and the ratio to GAPDH expression calculated (B), as described in Materials and Methods. The correlations of PAK1 or PAK4 expression to the expression of CK19, $\alpha$-SMA, palladin, GFAP or desmin were assessed by regression analysis, and $\mathrm{R}^{2}$ and $\mathrm{p}$ values are presented (C). pPAK1, phosphorylated active PAK1; tPAK1, total PAK1; pPAK4, phosphorylated active PAK4; tPAK4, total PAK4.

PF-3758309 inhibited HIF-1 $\alpha$ protein expression and reduced the expression of palladin and $\alpha$-SMA in TKCC cell lines

Since these cells were growing under hypoxia $\left(5 \% \mathrm{O}_{2}\right)$, the effect of PF-3758309 on the protein expression of HIF-1 $\alpha$ which is necessary for cell survival under hypoxia, was examined. After $48 \mathrm{~h}$ treatment with PF-3758309 at concentrations round the $\mathrm{IC}_{50}$ determined in a proliferation assay (Table 1), PF-3758309 significantly inhibited the expression of HIF-1 $\alpha$ protein in all six TKCC lines examined (Fig.2A\&B), decreased the expression of palladin protein in the TKCC 15, 18 and 2.1 lines, and decreased the expression of $\alpha$-SMA protein in the TKCC 15 and 26 lines (Fig.2A, C\&D). At the same time, PF-3758309 also inhibited the activities of PAK1 (Fig. 3A\&B) and PAK4 (Fig. 3A\&D), but not PAK2 (Supplementary Fig.1). These data indicate that inhibition of PAK1 and/or PAK4 by PF-3758309 down-regulated HIF- $1 \alpha$, and in some cases palladin and $\alpha$-SMA expression. 
4 of 15

A

$\begin{array}{lllllll}\text { TKCC } & 15 & 18 & 2.1 & 22 & 23 & 26\end{array}$ PF-3758309 - + - + - + - + + + +

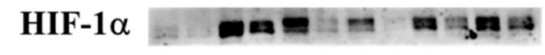
palladin - - - - =- - - - $\alpha-$ SMA - - - - - - GAPDH

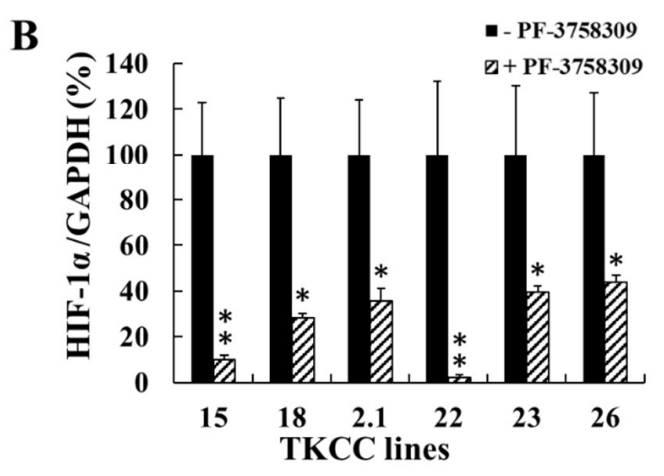

C

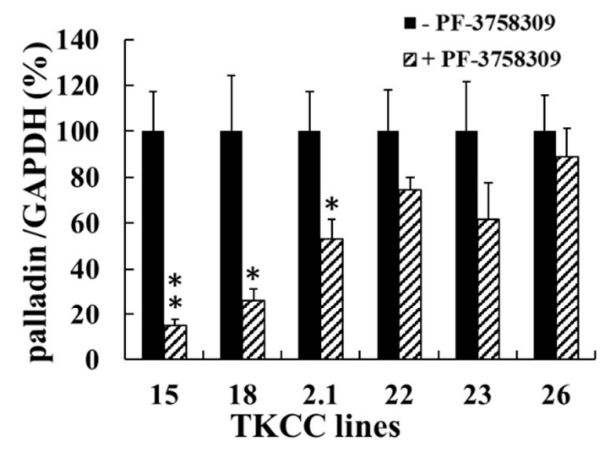

D

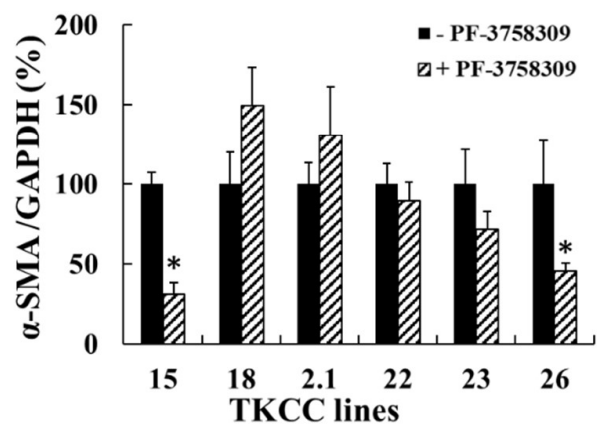

Fig.2. PF-3758309 inhibited the protein expression of HIF-1 $\alpha, \alpha$-SMA and palladin. The six patientderived pancreatic cancer cell lines were incubated with PF-3758309 at the concentrations listed in Table 1 in $5 \% \mathrm{O}_{2}$ for $48 \mathrm{~h}$. The protein levels of HIF- $1 \alpha, \alpha$-SMA and palladin were determined by Western blot (A). Relative protein expression was calculated by ratio to GAPDH, and the values for untreated cells were taken as $100 \%$ (B-D). The data were summarized from three independent experiments for the protein expression of HIF- $1 \alpha$ (B), $\alpha$-SMA (D) and palladin (C). ${ }^{*}, \mathrm{p}<0.05,{ }^{* *}, \mathrm{p}<0.01$, compared to the values obtained from non-PF-3758309-treated cells.
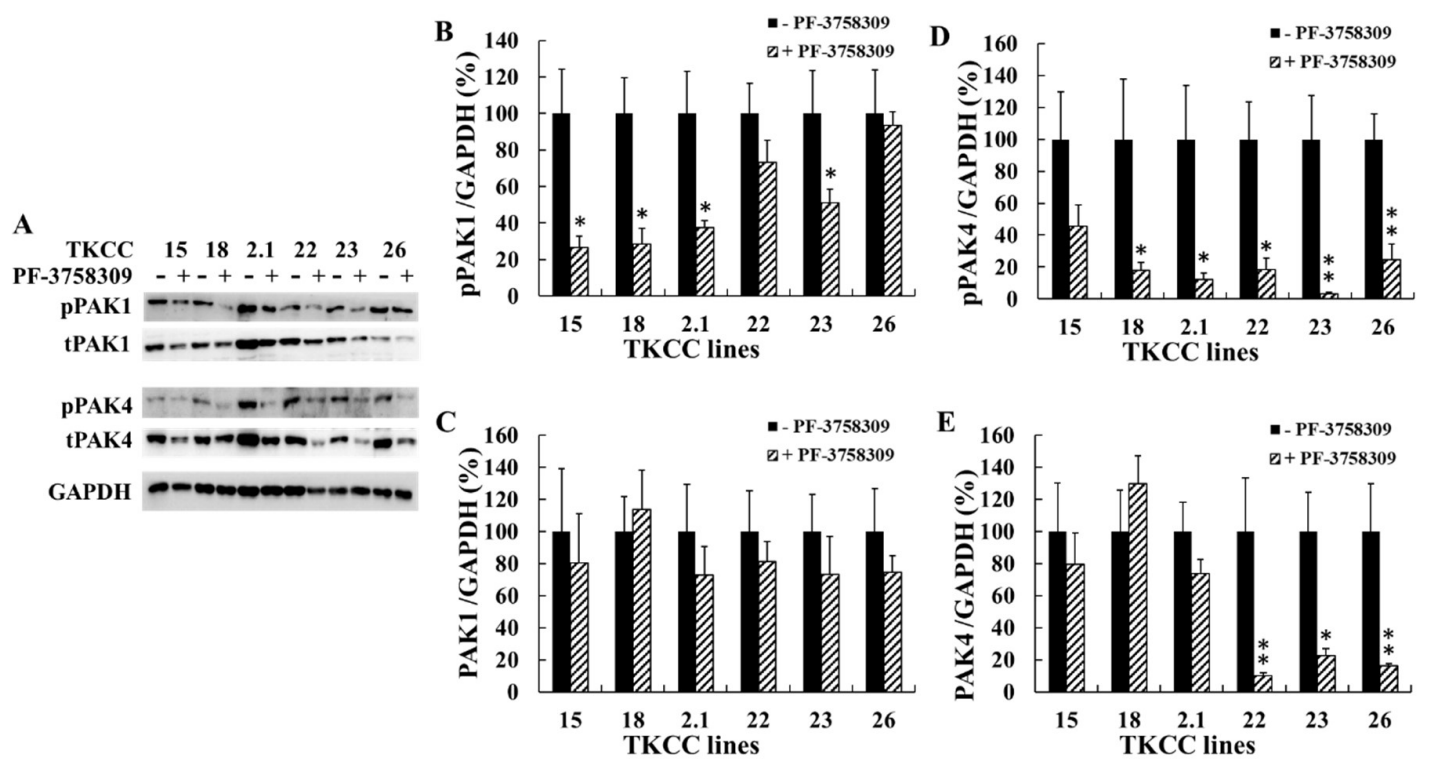

Fig.3. PF-3758309 suppressed the activities of PAK1 and PAK4. The six patient-derived pancreatic cancer cell lines were incubated with PF-3758309 as described in Fig.2. The protein levels of active phosphorylated PAK1 (pPAK1) and PAK4 (pPAK4), and total PAK1 (tPAK1) and PAK4 (tPAK4) were determined by Western blot. Relative protein expression was calculated by ratio to GAPDH, and the values for untreated cells were taken as $100 \%$ (B-E). The data were summarized from three 
independent experiments for the protein expression of pPAK1 (B), tPAK1 (C), pPAK4 (D) and tPAK4 (E). ${ }^{*}, \mathrm{p}<0.05,{ }^{* *}, \mathrm{p}<0.01$, compared to the values obtained from non-PF-3758309-treated cells.

PF-3758309 enhanced the inhibitory effects of 5-FU, gemcitabine and abraxane on TKCC cells in vitro.

The TKCC cells were first incubated with different concentrations of PF-3758309 for $48 \mathrm{~h}$ in $5 \%$ $\mathrm{O}_{2}$. PF-3758309 dose-dependently inhibited the proliferation of all TKCC lines examined (data not shown). The IC50 values of PF-3758309 for each cell line were calculated based on the proliferation assay and are listed in Table 1 . Similarly the $\mathrm{IC}_{50}$ values for 5-FU, gemcitabine and abraxane were also determined and are listed in Table 1. Additionally the TKCC cells were pre-treated with PF-3758309 at concentrations around the IC $\mathrm{C}_{50}$ values (Table 1 ) for $48 \mathrm{~h}$, followed by another $48 \mathrm{~h}$ treatment with 5$\mathrm{FU}$, gemcitabine or abraxane at concentrations around the IC50 values (Table 1) obtained in the absence of PF-3758309. PF-3758309 pre-treatment enhanced the inhibitory effects of 5-FU on TKCC $15,18,22,23$ and 26 cells, of gemcitabine on TKCC 18, 2.1, 22, 23 and 26 cells, and of abraxane on TKCC 15, 2.1, 22, 23 and 26 cells (Fig. 4) compared to each individual reagents alone. The combination of PF-3758309 with 5-FU further inhibited the proliferation of TKCC 15, 18 and 22 cells (Fig. 4A, 4B and 4D) compared to either PF-3758309 or 5-FU alone. The combination of PF-3758309 with gemcitabine further suppressed the proliferation of TKCC 18 and 22 cells (Fig. 4B\&D) compared to either PF-3758309 or gemcitabine alone. The combination of PF-3758309 with abraxane further reduced the proliferation of TKCC 15 (Fig. 4A) and TKCC 22 cells (Fig. 4D) compared to either PF3758309 or abraxane alone. These results indicate that PF-3758309 inhibited pancreatic cancer growth synergistically with multiple chemotherapeutic reagents.

\section{Table 1. IC50 values for a panel of patient-derived PDA cell lines}

\begin{tabular}{|c|c|c|c|c|}
\hline & PF-3758309 $(\mathrm{nM})$ & $5-\mathrm{FU}(\mu \mathrm{M})$ & Gemcitabine (nM) & Abraxane (nM) \\
\hline TKCC 15 & 5 & 10 & 10 & 10 \\
\hline TKCC 18 & 5 & 5 & 20 & 25 \\
\hline TKCC 2.1 & 400 & 5 & 5 & 15 \\
\hline TKCC 22 & 400 & 100 & 60 & $40 \mu M$ \\
\hline TKCC 23 & 200 & 150 & $1300(\mu M)$ & 70 \\
\hline TKCC 26 & 5 & 150 & 45 & $10 \mu M$ \\
\hline
\end{tabular}


A

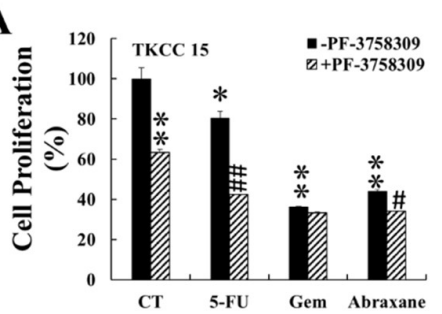

D

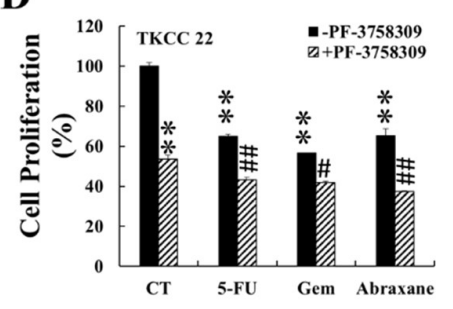

B

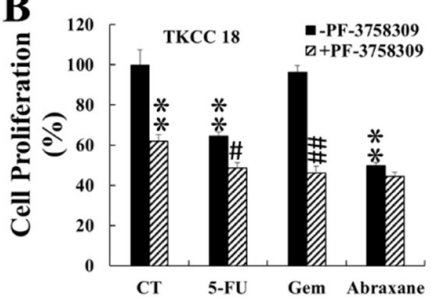

E

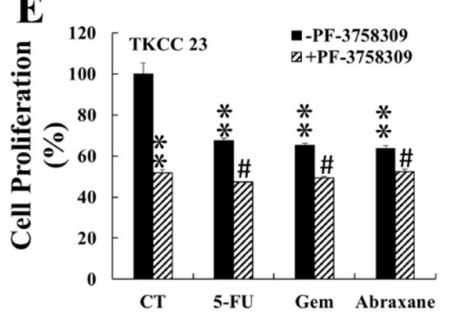

C

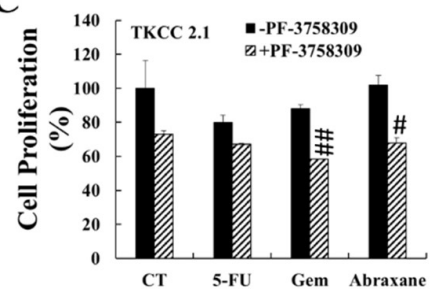

$\mathbf{F}$

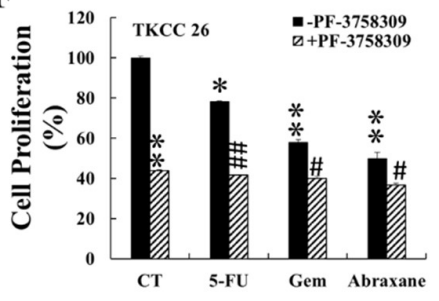

Fig.4. PF-3758309 enhanced the inhibitory effects of 5-FU, gemcitabine and abraxane on TKCC cell lines. Six TKCC cell lines (TKCC 15 (A); TKCC 18 (B); TKCC 2.1 (C); TKCC 22 (D); TKCC 23 (E); TKCC 26 (F)) were pre-incubated with PF-3758309 at the IC50 concentrations listed in Table 1 for 48h, followed by another $48 \mathrm{~h}$ incubation with 5-FU, gemcitabine (Gem) or abraxane at the $\mathrm{IC}_{50}$ concentrations listed in Table 1. Cell proliferation was determined as described in Materials and Methods. The values obtained from controls (CT) without any treatment were taken as $100 \%$. The data were summarized from at least three independent experiments. ${ }^{*}, \mathrm{p}<0.05,{ }^{* *}, \mathrm{p}<0.01$, compared to the values obtained for the controls of non-PF-3758309 pre-treated cells. \#, $\mathrm{p}<0.05, \# \#, \mathrm{p}<0.01$, compared to the values obtained from non-PF-3758309 pre-treated cells.

The combination of PF-3758309 with gemcitabine maximally inhibited tumour growth in vivo by suppressing proliferation.

SCID mice bearing TKCC 15 xenografted tumours were divided into the following five groups: control, PF-3758309, gemcitabine, PF-3758309 plus gemcitabine, and gemcitabine plus abraxane and the mice were treated as shown in Fig. 5A. At the beginning of the treatments (day 25), the tumour volumes (measured in $\mathrm{mm}^{3}$ ) were $72 \pm 12,82 \pm 11,82 \pm 11,80 \pm 13$, and $83 \pm 13$ for control, PF-3758309, gemcitabine, $\mathrm{PF}-3758309$ plus gemcitabine, and gemcitabine plus abraxane, respectively. The tumour volumes after treatment were reduced in all treatment groups compared to control (Fig. 5B). The combined treatment of PF-3758309 with gemcitabine significantly reduced the tumour volume from day 4 after starting the treatment. Compared to PF-3758309 alone, all combination treatments (PF3758309 plus gemcitabine or gemcitabine plus abraxane) significantly further inhibited the tumour volumes (Fig. 5B\&D). Compared to gemcitabine alone, the combination treatments of PF-3758309 plus gemcitabine and gemcitabine plus abraxane, significantly further reduced the tumour volumes (Fig. 5B\&D). Among the combination treatments, PF-3758309 plus gemcitabine inhibited the tumour volume maximally. All treated groups except for the PF-3758309 group showed significantly decreased tumour weights compared to control. However unlike the tumour volume, the tumour weight in the combination treatments did not show any further decrement compared to the single reagent treatment (Fig. 5C). 

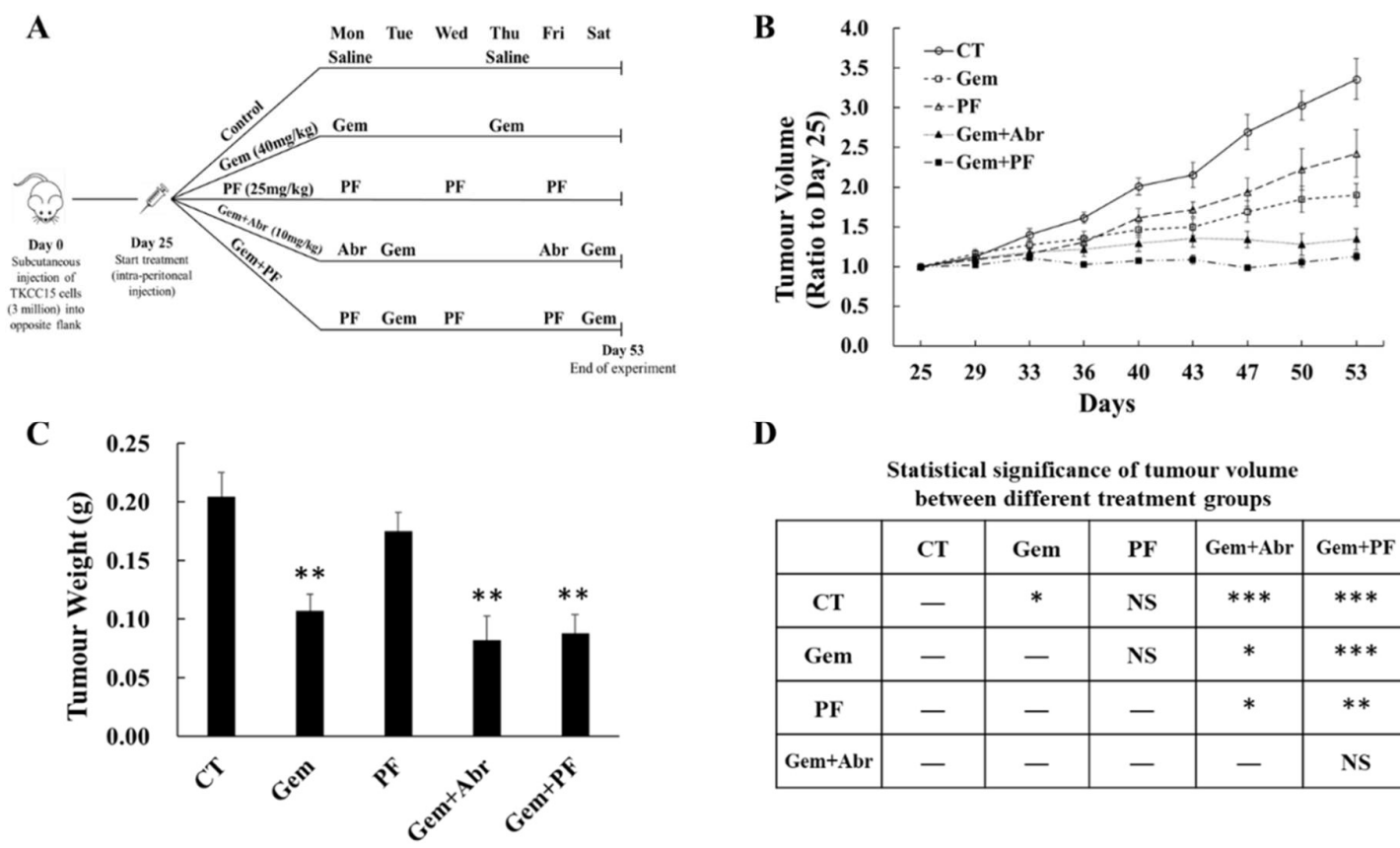

D

Statistical significance of tumour volume
between different treatment groups
\begin{tabular}{|c|c|c|c|c|c|}
\hline & CT & Gem & PF & Gem+Abr & Gem+PF \\
\hline CT & - & $*$ & NS & $* * *$ & $* * *$ \\
\hline Gem & - & - & NS & $*$ & $* * *$ \\
\hline PF & - & - & - & $*$ & $* *$ \\
\hline Gem+Abr & - & - & - & - & NS \\
\hline
\end{tabular}

Fig.5. PF-3758309 plus gemcitabine maximally inhibited tumour growth in vivo. TKCC 15 cells $\left(3 \times 10^{6} / 100 \mu \mathrm{l}\right)$ were injected subcutaneously into both flanks of 8 week old male SCID mice. At day 25 when the xenografted tumours had grown to around $80 \mathrm{~mm}^{3}$ in volume, the mice were randomly divided into the following 5 treatment groups (A): control (CT), PF-3758309 (PF), gemcitabine (Gem), gemcitabine plus PF-3758309 (Gem+PF), and Gem plus abraxane (Abr). Each group contained 3 mice bearing 2 tumours each, making 6 tumours in total. The treatments were given by intraperitoneal (i.p.) injection for four weeks, and the mice were culled on day 53 . The tumour weight $(C, * *, p<0.01$, compared to control) and volume (B) were measured and calculated as described in Material and Methods. The average values of tumour volumes in each group on day 25 (given in the Results section) were taken as 1 (B). The statistical significance (represented by $\mathrm{p}$ values) of tumour volumes between different groups were presented in D. In all cases the volumes thereafter were significantly different. NS = not significant.

Proliferation and apoptosis within xenograft tumour were determined by immunohistochemistry staining of Ki67 and cleaved caspase 3, respectively. Gemcitabine alone, not PF-3758309 alone, significantly inhibited cell proliferation to $83 \%$ of control. Combination treatment further reduced proliferation to $74 \%$ and $67 \%$ of control for gemcitabine plus abraxane and gemcitabine plus PF-3758309, respectively, and the reductions were significantly greater than that of gemcitabine alone treatment (Fig. 6 A\&B). There was no significant difference in apoptosis in all four treatment groups compared to control (Fig. $6 \mathrm{C} \& D$ ). These results indicated that combination of gemcitabine with PF-3758309 synergistically reduced tumour growth via suppressing tumour cell proliferation. This combination could reach an equal or even greater therapeutic efficacy compared to gemcitabine plus abraxane. 
A

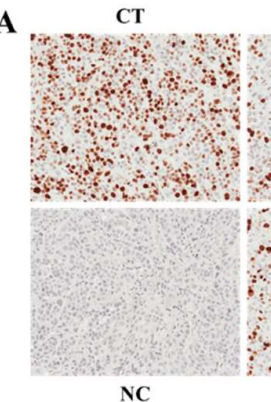

Gem

$\sin :$
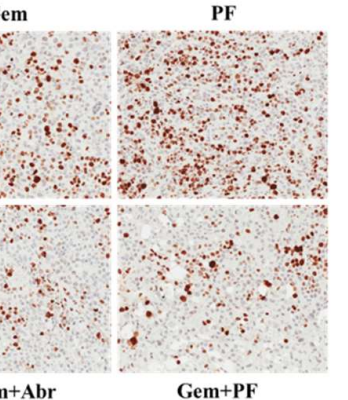

B

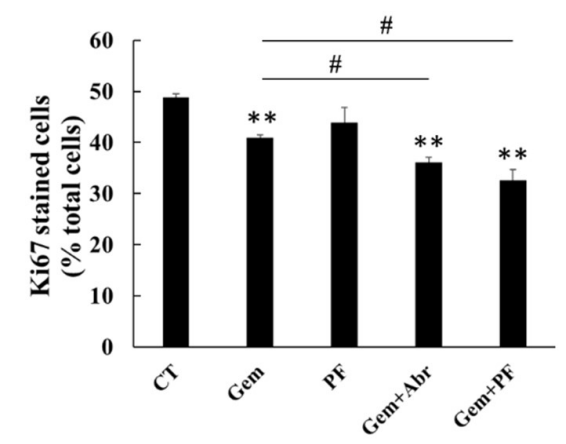

C

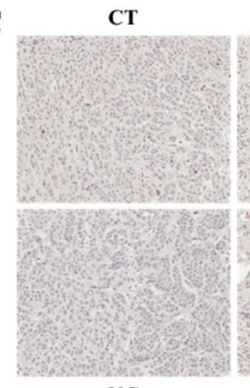

NC
Gem

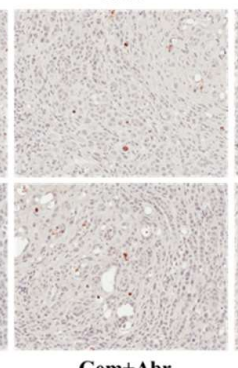

Gem+Abr

D

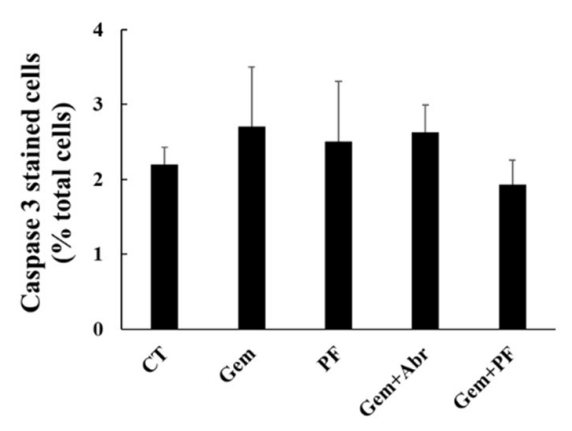

Fig.6. The combination of PF-3758309 and gemcitabine inhibited tumour cell proliferation in vivo. The xenograft tumour tissues from the experiments described in Fig. 5 were immunohistochemically stained with Ki67 (A\&B) and active cleaved caspapse $3(\mathrm{C} \& \mathrm{D})$ to measure proliferation and apoptosis respectively. The samples were analyzed as descibed in the Materials and Methods. NC: negative control stained without the primary antibody. CT: control, Gem: gemcitabine, PF: PF-3758309, Abr: Abraxane; ${ }^{* *}, \mathrm{p}<0.01$ compared to control, \#,p<0.05 compared to gemcitabine alone.

\section{$\alpha$-SMA and CK19 co-localized in cancer cells in vivo}

Consistent with the in vitro observation where the expression of $\alpha$-SMA was detected by both Western blots (Fig. 1) and immune staining in two TKCC cell lines (Fig.7A), the expression of $\alpha$-SMA was also detected in the tumours that originated from the xenografts of cell line TKCC15 (Fig.7B). Interestingly the co-expression and co-localisation of CK19 and $\alpha$-SMA was also found in some cancer cells of the xenograft tumours, particularly in the interface between tumour and stroma (Fig.7) regardless the different treatments. This observation suggests that those cells with co-expression and co-localisation of CK19 and $\alpha$-SMA might play important roles in local invasion and/or metastasis through the interaction of tumour with stroma. 
A

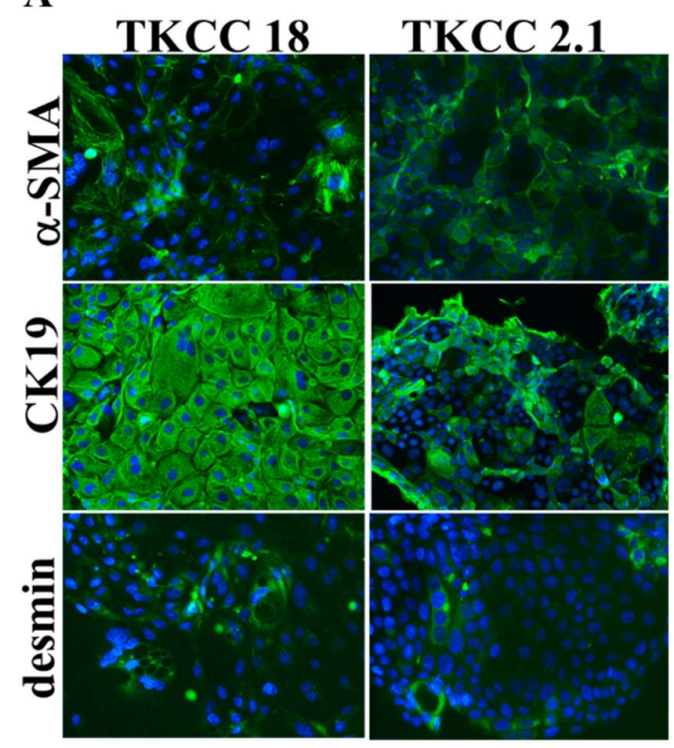

B

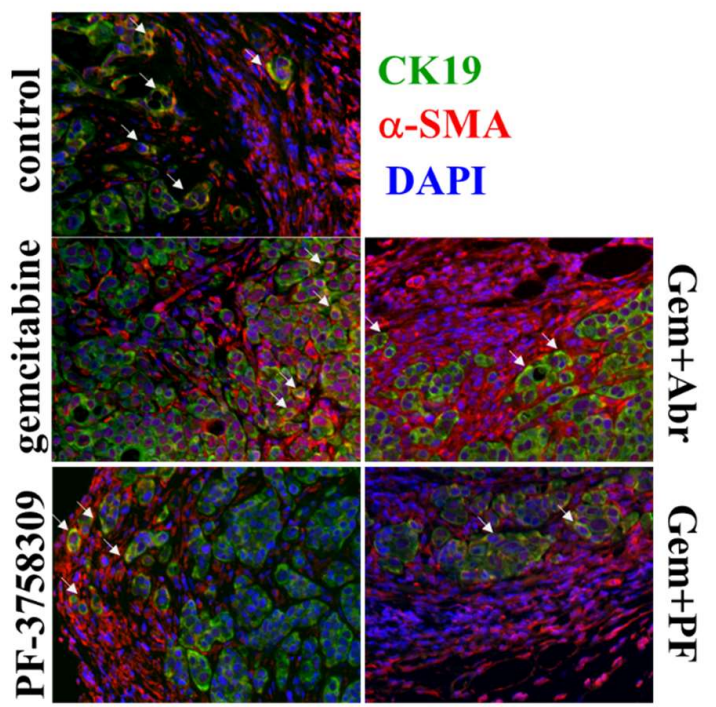

Fig.7. Intracellular localisation of CK19, $\alpha$-SMA and desmin (A). The intracellular localisations of CK19, $\alpha$-SMA and desmin were determined by immunofluorescent staining as described in Materials and Methods. The images were taken with a fluorescent microscope at a magnification of 40 times. Both CK19 and $\alpha$-SMA are located in the cytoplasm and membranes while desmin is in the cytoplasm only.

CK19 and $\alpha$-SMA co-localized in pancreatic cancer cells in vivo (B) . The tumour tissues generated from TKCC 15 xenografts were dissected from the mice treated with different reagents as shown in Fig. 5A, and double-stained with antibodies against CK19 (green) and $\alpha$-SMA (red). The photos were taken under a fluorescent microscopy at 20 times amplification. The arrows indicated the doublestained cells. Gem: gemcitabine, Abr: Abraxane, PF: PF-3758309.

\section{Discussion}

The data presented here indicates that the pan-PAK inhibitor PF-3758309 [21] is an attractive reagent for treatment of pancreatic cancer. In a panel of established cell lines derived from patients with pancreatic cancer [22], PF-3758309 not only inhibited cell proliferation on its own, but also enhanced the inhibitory effects of multiple chemotherapeutic reagents, including 5-FU, gemcitabine and abraxane. PF-3758309 also inhibited the protein expression of HIF-1 $\alpha$, palladin and $\alpha$-SMA by reducing the kinase activities of PAK1 and PAK4, which both play important roles in PDA progression. Our data showing that PF-3758309 stimulated the inhibitory effects of multiple chemotherapeutic reagents on PDA cells in vitro and in vivo, make PF-3758309 a potential reagent in adjunct or combination therapy in the treatment of pancreatic cancer. The combination of PF-3758309 with gemcitabine inhibited the xenograft growth of TKCC cells in mice to a level comparable to the combination of gemcitabine with abraxane. This finding indicates that gemcitabine plus PF-3758309 could be used as an alternative therapy, with lower toxicity, than gemcitabine plus abraxane, which is currently used as a front-line chemotherapy for pancreatic cancer.

TKCC cells express not only the cancer cell marker CK19, but also stromal cell markers including palladin, $\alpha$-SMA, desmin and GFAP. Both PAK1 and PAK4 were also expressed in these cell lines. Interestingly, the expression of PAK1 was significantly correlated with CK19, while the expression of PAK4 was significantly correlated with palladin, $\alpha$-SMA, and GFAP (Fig.1C). This result suggests that PAK1 and PAK4 play divergent roles in the regulation or maintenance of an "epithelial" or 
"mesenchymal"-like state in cancer progression, and is consistent with a recent report that expression of PAK1 and PAK4 is associated with different outcomes in pancreatic cancer patients [23]. PAK1 and PAK4 also play different roles in pancreatic function, as PAK1 depletion causes deficits in glucose clearance [24], while PAK4 depletion does not alter pancreas development or function [25].

Inhibition of PAKs by PF-3758309 in TKCC cell lines significantly decreased the expression of several key proteins implicated in cancer cell survival and cytoskeletal reorganization. The observation that PF-3758309 significantly decreased the expression of HIF-1 $\alpha$ in all six TKCC lines suggests critical roles for PAK1 and/or PAK4 in the survival and growth of these cancer cells under hypoxia. Inhibition of PAK1 and PAK4 by PF-3758309 also suppressed the expression of palladin in three of these TKCC lines. Palladin stimulates invasion of pancreatic cancer cells by promoting invadopodia formation in cancer-associated fibroblasts [26]. Tissue microarrays of resected PDA samples from 167 patients have shown that expression of palladin is correlated with a significantly lower disease-specific survival rate [6]. Therefore, inhibition of palladin would be expected to suppress pancreatic cancer metastasis and promote patients' survival. $\alpha$-SMA is an established marker for PSC activation [27], and its expression is negatively correlated with PDA survival [28]. We have previously reported that inhibition of PAK1 decreased proliferation and increased apoptosis of PSCs while suppressing $\alpha$-SMA expression in PSCs [12]. Here for the first time we have detected the expression of $\alpha$-SMA by both Western blot and immunofluorescent staining in cells established from xenografts isolated from pancreatic cancer patients. PF-3758309 significantly reduced the protein expression of $\alpha$-SMA in two out of the six pancreatic cancer cell lines. The different effect of PF-3758309 on $\alpha$-SMA expression in these cancer cell lines may be due to the heterogeneous nature of pancreatic cancers. Out findings that both CK19 and $\alpha$-SMA co-expressed and co-localized in some cancer cells in the xenograft tumours, in particularly that these cancer cells with co-localisation of CK19 and $\alpha$-SMA distributed at the interface between tumour and stroma, suggested important roles of those cells in local invasion and/or metastasis through the interaction of tumour with stroma.

\section{Materials and Methods}

\section{Cell Proliferation}

The patient-derived cell lines (TKCC 2.1, TKCC15, TKCC18, TKCC22, TKCC23, TKCC26) used in this study were isolated at $\underline{T}$ he Kinghorn $\underline{\text { Cancer }}$ Centre (TKCC) from primary patient-derived pancreatic ductal adenocarcinoma xenografts [29] and cultured as previously described [22]. 5x103 cells/well were incubated with different concentrations of PF-3758309 for 48h or pre-treated with PF3758309 at the IC50 calculated for proliferation (Table 1) for $48 \mathrm{~h}$, followed by treatment with gemcitabine, 5-FU or Abraxane for another 48h in the absence of PF-3758309. Cell proliferation was measured using a CyQUANT Direct Cell Proliferation Assay Kit (Thermo Fisher, Melbourne, Australia) according to the manufacturer's instruction. Fluorescence intensity was determined using a plate reader (FLUOstar OPTIMA, BMG Labtech, Melbourne, Australia).

\section{Mouse study}

All mouse experiments were approved by the Austin Health Animal Ethics Committee (A2015/05269). SCID mice were purchased from the Animal Resource Centre (Perth, Australia). TKCC 15 cells $\left(3 \times 10^{6}\right.$ cells $/ 100 \mu \mathrm{l} /$ site) were injected subcutaneously into the flanks of 8 weeks old SCID mice. On day 25 after subcutaneous injection, the mice were divided into 5 groups: control (saline), gemcitabine $(40 \mathrm{mg} / \mathrm{Kg}$, dissolved in saline), PF-3758309 (25 mg/kg, dissolved in 5\% DMSO in saline) alone, PF-3758309 plus gemcitabine, and gemcitabine plus abraxane (10 mg/Kg, dissolved in saline). The mice were given these treatments by intraperitoneal injection (i.p.) as shown in Fig. 5A for four weeks. Tumour growth was monitored as previously described [30]. Tumour volume was calculated by using the formula: $\mathrm{V}=\mathrm{L}^{*} \mathrm{~W}^{*} \mathrm{H}^{*}(\pi / 6)$, where $\mathrm{L}$ was the length; $\mathrm{W}$ the width; and $\mathrm{H}$ the height. 
Tumour volumes on day 25 in each group were taken as 1 . At the end of the experiment mice were culled, and tumours were dissected and divided into 2 parts, of which one was frozen for tissue extraction, and the other was fixed in 10\% formalin and then paraffin-imbedded for immune-staining.

\section{Western blot}

TKCC cells were cultured to $80 \%$ confluence and lysed in SDS sample buffer. Protein expression profiles were determined by Western blot using antibodies against the following proteins: total and phosphorylated (active) PAK1, PAK2 and PAK4 (Cell Signaling Technology, Arundel, Australia), cytokeratin 19 (CK19), a-SMA, palladin, GFAP (Abcam, Cambridge, MA), desmin (Santa Cruz Biotechnology, Taxas, USA), and GAPDH (Cell Signaling Technology, Arundel, Australia). Cell lysates were also prepared from TKCC cells after treatment with PF-3758309 for 48h, and protein expression was measured by Western blot using antibodies against HIF-1 $\alpha$ (BD Biosciences, North Ryde, Australia), total and phosphorylated PAK1, PAK2 and PAK4, $\alpha-S M A$, palladin and GAPDH. The relative amount of each protein was calculated as the ratio of the density of its band to the density of the GAPDH band. The correlation of PAK1 or PAK4 expression with CK19, $\alpha$-SMA, palladin, GFAP and desmin expression was determined by regression analysis using Sigma Plot 12 (Systat Software Inc., California, USA).

\section{Immunofluorescent staining}

TKCC cells were grown on sterilized glass slides to $80 \%$ confluency. After fixation with $4 \%$ paraformaldehyde for $5 \mathrm{~min}$, cells were permeabilized with $0.1 \%$ Triton X-100 for 5 min at room temperature and blocked with blocking buffer $5 \%$ normal goat serum in 1\% BSA in TBST (Trisbuffered saline, 0.1\% Tween 20)) for 1 hour. Cells were stained with antibodies against CK19 (1:300), $\alpha$-SMA (1:300) and desmin (1:300) in blocking buffer overnight at $4^{\circ} \mathrm{C}$, followed by incubation with secondary antibodies conjugated with AlexaFlour 488 (1:1000) in 1\% BSA in TBST for 1 h at room temperature in the dark. Cells were counterstained with DAPI (4', 6-diamidino-2-phenylindole dihydrochloride, Life Technologies, Scoresby, Australia) (1:5000) in PBS for 5 min. Coverslips were mounted using fluorescence mounting medium. The samples were observed and photographed using an Evos cell image system (Life Technologies). For double staining of CK19 and $\alpha$-SMA in tumour tissues, an anti- $\alpha$-SMA antibody was purchased from Sigma and the images were taken under a fluorescent microscope (Eclipse Ti-E Model, Nikon) at 20 times amplification.

\section{Immunohistochemistry}

Tumour tissues, collected from the animal model as described above, were stained with Ki67 antibody (Thermo Fisher Scientific, Australia) for proliferation, cleaved caspase 3 antibody (Cell Signalling Technology, Arundel, Australia) for apoptosis. Samples were imaged using a Leica microscope for at least 10 fields at 40 times magnification. For quantification, the ratio of positive cells to total number of cells in each field was calculated.

\section{Statistical analysis}

All values are expressed as mean \pm standard error. The in vitro data are from three independent experiments. The in vivo data were collated according to numbers of tumour samples. Data were analyzed by one-way ANOVA or student's t-test (SPSS, IBM, New York, NY). Differences between two means with $\mathrm{p}<0.05$ were considered significant.

\section{Conclusions}

In summary we have demonstrated here that the combination of the PAK inhibitor PF3758309 with multiple chemotherapeutic reagents further suppressed the growth of a panel of pancreatic cancer cells derived from patients' samples compared to the chemotherapeutic reagents on their own. 
The combination of PF-3758309 with gemcitabine showed maximal inhibition of the pancreatic cancer cells in vivo. The extent of inhibition was comparable to that achieved with gemcitabine plus abraxane. The combination of PF-3758309 with gemcitabine therefore provides a potential treatment option with less toxicity.

Author Contributions: $\mathrm{HH}, \mathrm{GSB}$ and MN conceived and designed the study; $\mathrm{NH}, \mathrm{KW}, \mathrm{XW}, \mathrm{MP}, \mathrm{AP}$, $\mathrm{JM}$ and $\mathrm{HH}$ acquired, analysed and interpreted the data; $\mathrm{HH}$ drafted the manuscript; $\mathrm{NH}, \mathrm{KW}, \mathrm{XW}$, GSB, MP, AP, JM, MN and HH reviewed and revised the manuscript. All authors have read and approved the final version of this manuscript

Funding: This work was supported by grants from the Pancare foundation. Dr Kai Wang is supported by scholarships from the University of Melbourne and Pancare foundation (Moshe Sambor Scholarship).

Acknowledgments: We thank Dr Dannel Yeo for his help and suggestion in TKCC cell culture.

Conflicts of Interest: The authors declare no conflict of interest.

\section{Abbreviations}

$\begin{array}{ll}\text { PAK1 } & \text { P21-activated kinase 1 } \\ \text { PAK4 } & \text { P21-activated kinase 4 } \\ \text { PDA } & \text { pancreatic ductal adenocarcinoma } \\ \text { 5-FU } & 5 \text {-fluorouracil } \\ \text { CK19 } & \text { cytokeratin 19 } \\ \text { HIF- } \alpha & \text { hypoxia-inducible factor 1 } \alpha \\ \text { GFAP } & \text { glial fibrillary acidic protein } \\ \text { PSCs } & \text { pancreatic stellate cells } \\ \alpha \text {-SMA } & \alpha \text {-smooth muscle actin }\end{array}$

\section{Appendix A}

A

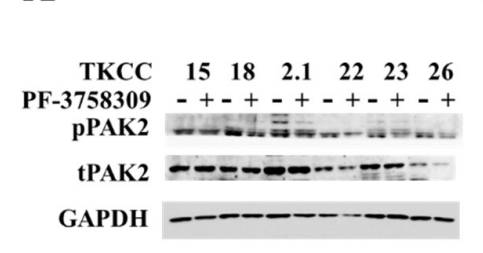

B

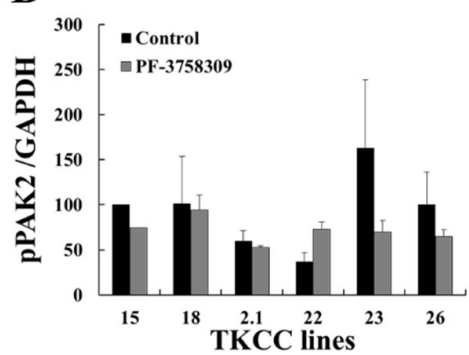

C

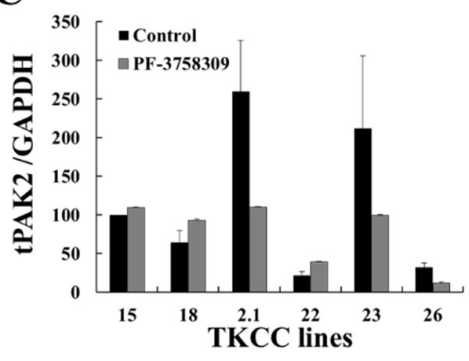

Supplementary Fig.1. PF-3758309 had no effect on the activity or expression of PAK2. Six TKCC cell lines were treated with PF-3758309 at the IC50 concentrations calculated from proliferation data (Table 1). After $48 \mathrm{~h}$, culture medium was removed, the cells were lysed and the cell lysates were subjected to immune-blotting using antibodies against phosphorylated PAK2 (pPAK2), total PAK2 (tPAK2) and GAPDH.

\section{Appendix B}

All appendix sections must be cited in the main text. In the appendixes, Figures, Tables, etc. should be labeled starting with 'A', e.g., Figure A1, Figure A2, etc. 
13 of 15

\section{References}

1. Lau, S. C.; Cheung, W. Y., Evolving treatment landscape for early and advanced pancreatic cancer. World J Gastrointest Oncol 2017, 9, (7), 281-292.

2. Conroy, T.; Desseigne, F.; Ychou, M.; Bouche, O.; Guimbaud, R.; Becouarn, Y.; Adenis, A.; Raoul, J. L.; Gourgou-Bourgade, S.; de la Fouchardiere, C.; Bennouna, J.; Bachet, J. B.; Khemissa-Akouz, F.; Pere-Verge, D.; Delbaldo, C.; Assenat, E.; Chauffert, B.; Michel, P.; Montoto-Grillot, C.; Ducreux, M.; Groupe Tumeurs Digestives of, U.; Intergroup, P., FOLFIRINOX versus gemcitabine for metastatic pancreatic cancer. N Engl J Med 2011, 364, (19), 1817-25.

3. Von Hoff, D. D.; Ervin, T.; Arena, F. P.; Chiorean, E. G.; Infante, J.; Moore, M.; Seay, T.; Tjulandin, S. A.; Ma, W. W.; Saleh, M. N.; Harris, M.; Reni, M.; Dowden, S.; Laheru, D.; Bahary, N.; Ramanathan, R. K.; Tabernero, J.; Hidalgo, M.; Goldstein, D.; Van Cutsem, E.; Wei, X.; Iglesias, J.; Renschler, M. F., Increased survival in pancreatic cancer with nabpaclitaxel plus gemcitabine. N Engl J Med 2013, 369, (18), 1691-703.

4. Neesse, A.; Michl, P.; Frese, K. K.; Feig, C.; Cook, N.; Jacobetz, M. A.; Lolkema, M. P.; Buchholz, M.; Olive, K. P.; Gress, T. M.; Tuveson, D. A., Stromal biology and therapy in pancreatic cancer. Gut 2011, 60, (6), 861-8.

5. Apte, M. V.; Pirola, R. C.; Wilson, J. S., Pancreatic stellate cells: a starring role in normal and diseased pancreas. Front Physiol 2012, 3, 344.

6. Sato, D.; Tsuchikawa, T.; Mitsuhashi, T.; Hatanaka, Y.; Marukawa, K.; Morooka, A.; Nakamura, T.; Shichinohe, T.; Matsuno, Y.; Hirano, S., Stromal Palladin Expression Is an Independent Prognostic Factor in Pancreatic Ductal Adenocarcinoma. PLoS One 2016, 11, (3), e0152523.

7. Zhou, W.; Jubb, A. M.; Lyle, K.; Xiao, Q.; Ong, C. C.; Desai, R.; Fu, L.; Gnad, F.; Song, Q.; Haverty, P. M.; Aust, D.; Grutzmann, R.; Romero, M.; Totpal, K.; Neve, R. M.; Yan, Y.; Forrest, W. F.; Wang, Y.; Raja, R.; Pilarsky, C.; de Jesus-Acosta, A.; Belvin, M.; Friedman, L. S.; Merchant, M.; Jaffee, E. M.; Zheng, L.; Koeppen, H.; Hoeflich, K. P., PAK1 mediates pancreatic cancer cell migration and resistance to MET inhibition. J Pathol 2014, 234, (4), 502-13.

8. Mahlamaki, E. H.; Kauraniemi, P.; Monni, O.; Wolf, M.; Hautaniemi, S.; Kallioniemi, A., High-resolution genomic and expression profiling reveals 105 putative amplification target genes in pancreatic cancer. Neoplasia 2004, 6, (5), 432-9.

9. Yeo, D.; He, H.; Baldwin, G. S.; Nikfarjam, M., The role of p21-activated kinases in pancreatic cancer. Pancreas 2015, 44, (3), 363-9.

10. Yeo, D.; He, H.; Patel, O.; Lowy, A. M.; Baldwin, G. S.; Nikfarjam, M., FRAX597, a PAK1 inhibitor, synergistically reduces pancreatic cancer growth when combined with gemcitabine. BMC Cancer 2016, 16, 24.

11. Jagadeeshan, S.; Subramanian, A.; Tentu, S.; Beesetti, S.; Singhal, M.; Raghavan, S.; Surabhi, R. P.; Mavuluri, J.; Bhoopalan, H.; Biswal, J.; Pitani, R. S.; Chidambaram, S.; Sundaram, S.; Malathi, R.; Jeyaraman, J.; Nair, A. S.; Venkatraman, G.; Rayala, S. K., P21-activated kinase 1 (Pak1) signaling influences therapeutic outcome in pancreatic cancer. Ann Oncol 2016, 27, (8), 1546-56. 
12. Yeo, D.; Phillips, P.; Baldwin, G. S.; He, H.; Nikfarjam, M., Inhibition of group 1 p21activated kinases suppresses pancreatic stellate cell activation and increases survival of mice with pancreatic cancer. Int J Cancer 2017, 140, (9), 2101-2111.

13. Tyagi, N.; Bhardwaj, A.; Singh, A. P.; McClellan, S.; Carter, J. E.; Singh, S., p-21 activated kinase 4 promotes proliferation and survival of pancreatic cancer cells through AKT- and ERK-dependent activation of NF-kappaB pathway. Oncotarget 2014, 5, (18), 8778-89.

14. Moon, S. U.; Kim, J. W.; Sung, J. H.; Kang, M. H.; Kim, S. H.; Chang, H.; Lee, J. O.; Kim, Y. J.; Lee, K. W.; Kim, J. H.; Bang, S. M.; Lee, J. S., p21-Activated Kinase 4 (PAK4) as a Predictive Marker of Gemcitabine Sensitivity in Pancreatic Cancer Cell Lines. Cancer Res Treat 2015, 47, (3), 501-8.

15. Mohammad, R. M.; Li, Y.; Muqbil, I.; Aboukameel, A.; Senapedis, W.; Baloglu, E.; Landesman, Y.; Philip, P. A.; Azmi, A. S., Targeting Rho GTPase effector p21 activated kinase 4 (PAK4) suppresses p-Bad-microRNA drug resistance axis leading to inhibition of pancreatic ductal adenocarcinoma proliferation. Small GTPases 2017, 0.

16. Aboukameel, A.; Muqbil, I.; Senapedis, W.; Baloglu, E.; Landesman, Y.; Shacham, S.; Kauffman, M.; Philip, P. A.; Mohammad, R. M.; Azmi, A. S., Novel p21-Activated Kinase 4 (PAK4) Allosteric Modulators Overcome Drug Resistance and Stemness in Pancreatic Ductal Adenocarcinoma. Mol Cancer Ther 2017, 16, (1), 76-87.

17. Murray, B. W.; Guo, C.; Piraino, J.; Westwick, J. K.; Zhang, C.; Lamerdin, J.; Dagostino, E.; Knighton, D.; Loi, C. M.; Zager, M.; Kraynov, E.; Popoff, I.; Christensen, J. G.; Martinez, R.; Kephart, S. E.; Marakovits, J.; Karlicek, S.; Bergqvist, S.; Smeal, T., Small-molecule p21activated kinase inhibitor PF-3758309 is a potent inhibitor of oncogenic signaling and tumor growth. Proc Natl Acad Sci U S A 2010, 107, (20), 9446-51.

18. Ong, C. C.; Jubb, A. M.; Jakubiak, D.; Zhou, W.; Rudolph, J.; Haverty, P. M.; Kowanetz, M.; Yan, Y.; Tremayne, J.; Lisle, R.; Harris, A. L.; Friedman, L. S.; Belvin, M.; Middleton, M. R.; Blackwood, E. M.; Koeppen, H.; Hoeflich, K. P., P21-activated kinase 1 (PAK1) as a therapeutic target in BRAF wild-type melanoma. J Natl Cancer Inst 2013, 105, (9), 606-7.

19. Ryu, B. J.; Lee, H.; Kim, S. H.; Heo, J. N.; Choi, S. W.; Yeon, J. T.; Lee, J.; Lee, J.; Cho, J. Y.; Kim, S. H.; Lee, S. Y., PF-3758309, p21-activated kinase 4 inhibitor, suppresses migration and invasion of A549 human lung cancer cells via regulation of CREB, NF-kappaB, and beta-catenin signalings. Mol Cell Biochem 2014, 389, (1-2), 69-77.

20. Huynh, N.; Shulkes, A.; Baldwin, G.; He, H., Up-regulation of stem cell markers by P21activated kinase 1 contributes to 5-fluorouracil resistance of colorectal cancer. Cancer Biol Ther 2016, 17, (8), 813-23.

21. Guo, C.; McAlpine, I.; Zhang, J.; Knighton, D. D.; Kephart, S.; Johnson, M. C.; Li, H.; Bouzida, D.; Yang, A.; Dong, L.; Marakovits, J.; Tikhe, J.; Richardson, P.; Guo, L. C.; Kania, R.; Edwards, M. P.; Kraynov, E.; Christensen, J.; Piraino, J.; Lee, J.; Dagostino, E.; DelCarmen, C.; Deng, Y. L.; Smeal, T.; Murray, B. W., Discovery of pyrroloaminopyrazoles as novel PAK inhibitors. J Med Chem 2012, 55, (10), 4728-39.

22. Chou, A.; Froio, D.; Nagrial, A. M.; Parkin, A.; Murphy, K. J.; Chin, V. T.; Wohl, D.; Steinmann, A.; Stark, R.; Drury, A.; Walters, S. N.; Vennin, C.; Burgess, A.; Pinese, M.; Chantrill, L. A.; Cowley, M. J.; Molloy, T. J.; Australian Pancreatic Cancer Genome, I.; Waddell, N.; Johns, A.; Grimmond, S. M.; Chang, D. K.; Biankin, A. V.; Sansom, O. J.; 
Morton, J. P.; Grey, S. T.; Cox, T. R.; Turchini, J.; Samra, J.; Clarke, S. J.; Timpson, P.; Gill, A. J.; Pajic, M., Tailored first-line and second-line CDK4-targeting treatment combinations in mouse models of pancreatic cancer. Gut 2017.

23. Park, S.; Kim, J. W.; Kim, H.; Kim, J. W.; Kim, Y. J.; Lee, K. W.; Kim, J. H.; Kim, J. H.; Hwang, J. H.; Choi, Y. R.; Cho, J. Y.; Yoon, Y. S.; Han, H. S., Prognostic value of p21activated kinase 4 in resected pancreatic cancer. APMIS 2017, 125, (8), 699-707.

24. Wang, Z.; Oh, E.; Clapp, D. W.; Chernoff, J.; Thurmond, D. C., Inhibition or ablation of p21activated kinase (PAK1) disrupts glucose homeostatic mechanisms in vivo. J Biol Chem 2011, 286, (48), 41359-67.

25. Zhao, M.; Rabieifar, P.; Costa, T. D. F.; Zhuang, T.; Minden, A.; Lohr, M.; Heuchel, R.; Stromblad, S., Pdx1-Cre-driven conditional gene depletion suggests PAK4 as dispensable for mouse pancreas development. Sci Rep 2017, 7, (1), 7031.

26. Goicoechea, S. M.; Garcia-Mata, R.; Staub, J.; Valdivia, A.; Sharek, L.; McCulloch, C. G.; Hwang, R. F.; Urrutia, R.; Yeh, J. J.; Kim, H. J.; Otey, C. A., Palladin promotes invasion of pancreatic cancer cells by enhancing invadopodia formation in cancer-associated fibroblasts. Oncogene 2014, 33, (10), 1265-73.

27. Han, S.; Delitto, D.; Zhang, D.; Sorenson, H. L.; Sarosi, G. A.; Thomas, R. M.; Behrns, K. E.; Wallet, S. M.; Trevino, J. G.; Hughes, S. J., Primary outgrowth cultures are a reliable source of human pancreatic stellate cells. Lab Invest 2015, 95, (11), 1331-40.

28. Erkan, M.; Michalski, C. W.; Rieder, S.; Reiser-Erkan, C.; Abiatari, I.; Kolb, A.; Giese, N. A.; Esposito, I.; Friess, H.; Kleeff, J., The activated stroma index is a novel and independent prognostic marker in pancreatic ductal adenocarcinoma. Clin Gastroenterol Hepatol 2008, 6, (10), 1155-61.

29. Waddell, N.; Pajic, M.; Patch, A. M.; Chang, D. K.; Kassahn, K. S.; Bailey, P.; Johns, A. L.; Miller, D.; Nones, K.; Quek, K.; Quinn, M. C.; Robertson, A. J.; Fadlullah, M. Z.; Bruxner, T. J.; Christ, A. N.; Harliwong, I.; Idrisoglu, S.; Manning, S.; Nourse, C.; Nourbakhsh, E.; Wani, S.; Wilson, P. J.; Markham, E.; Cloonan, N.; Anderson, M. J.; Fink, J. L.; Holmes, O.; Kazakoff, S. H.; Leonard, C.; Newell, F.; Poudel, B.; Song, S.; Taylor, D.; Waddell, N.; Wood, S.; Xu, Q.; Wu, J.; Pinese, M.; Cowley, M. J.; Lee, H. C.; Jones, M. D.; Nagrial, A. M.; Humphris, J.; Chantrill, L. A.; Chin, V.; Steinmann, A. M.; Mawson, A.; Humphrey, E. S.; Colvin, E. K.; Chou, A.; Scarlett, C. J.; Pinho, A. V.; Giry-Laterriere, M.; Rooman, I.; Samra, J. S.; Kench, J. G.; Pettitt, J. A.; Merrett, N. D.; Toon, C.; Epari, K.; Nguyen, N. Q.; Barbour, A.; Zeps, N.; Jamieson, N. B.; Graham, J. S.; Niclou, S. P.; Bjerkvig, R.; Grutzmann, R.; Aust, D.; Hruban, R. H.; Maitra, A.; Iacobuzio-Donahue, C. A.; Wolfgang, C. L.; Morgan, R. A.; Lawlor, R. T.; Corbo, V.; Bassi, C.; Falconi, M.; Zamboni, G.; Tortora, G.; Tempero, M. A.; Australian Pancreatic Cancer Genome, I.; Gill, A. J.; Eshleman, J. R.; Pilarsky, C.; Scarpa, A.; Musgrove, E. A.; Pearson, J. V.; Biankin, A. V.; Grimmond, S. M., Whole genomes redefine the mutational landscape of pancreatic cancer. Nature 2015, 518, (7540), 495-501.

30. Huynh, N.; Shulkes, A.; Baldwin, G.; He, H., Up-regulation of stem cell markers by P21activated kinase 1 contributes to 5-fluorouracil resistance of colorectal cancer. Cancer biology $\mathcal{E}$ therapy 2016, 1-11. 